\title{
Understanding the language of drugged plants
}

Vivek Halder and Eugenia Russinova

\section{Plant biologists have recognized the potential in using small molecules identified from chemical libraries to provide insights into biological questions relevant to plants. However, the classical genetics mindset still predominant among plant scientists should evolve to embrace cross-disciplinary chemical genetic projects that will benefit future plant research.}

Historically, chemistry and plants have been tightly linked because plants, as a source of highly diverse natural products, have inspired synthetic chemists and drug developers. Furthermore, the discovery of phytohormones and their receptor-mediated signaling pathways have piqued the interest of many plant biologists to search chemical space to identify functionally similar small bioactive compounds. Over the last two decades, the focus has shifted towards more targeted classical genetics research wherein plant biologists have rationally perturbed biological responses to address fundamental questions ${ }^{1}$.

The contribution of classical genetics towards the advancement of plant science is irrefutable, but the role of chemical genetics needs to be seriously considered as well ${ }^{1,2}$. Despite being a relatively young discipline, plant chemical genetics has already proven its potential to bridge a number of genotype-to-phenotype gaps observed in plant classical genetics ${ }^{1,2}$. Chemical genetics, which is a part of the much broader chemical biology theme, deals with the application of small molecules that can selectively modulate the function of a protein or of members of a protein family, resembling phenotypically (a) genetic mutation(s) of the protein-encoding gene ${ }^{3}$. When compared to the vast scientific literature in the drug discovery field, the scope of plant 
chemical genetics research is still relatively small but has begun to increase in recent years. At this stage, plant biologists need to consider the balance between traditional genetics and chemical genetics as approaches to probe mechanisms in plant biology (Fig. 1).

Arabidopsis thaliana (L.) Heynh. (Arabidopsis) has emerged as a leading model system for plant genetics studies, and offers many advantages for chemical genetics, including a wellannotated genome, ease of growth in multi-well plates and on various media, and availability of reporter systems, amongst others. Because classical genetics has difficulty overcoming issues of gene redundancy, lethality, and pleiotropy, small molecules offer a promising alternative, along with the added feature that chemical probes may be used to control function in a precise time frame or in a reversible or dose-dependent manner ${ }^{2}$.

The identification of abscisic acid (ABA) receptors of Arabidopsis represents a foundational contribution of plant chemical genetics wherein a phenotype-based (i.e., forward chemical genetics) screen resulted in the discovery of the small molecule pyrabactin ${ }^{4}$. When employed as a probe, pyrabactin was found to act as a selective agonist for the ABA receptor PYR1. Since that time, many laboratories have adopted chemical genetics screens to study endomembrane trafficking ${ }^{5}$, phytohormone signaling ${ }^{6-10}$, pathogen defense ${ }^{11}$, as well as plant cell wall modifications ${ }^{12,13}$, amongst several others. These examples provide ample demonstration of the complementarity of plant chemical genetics to traditional plant biology approaches.

Despite the growing interest in using chemical genetics in plant research, the number of plant biology labs applying chemical genetics remains low and still faces many technological hurdles. Here we explore these challenges and discuss how plant chemical genetics may be advanced by adopting chemical biology approaches that have matured from their use in other 
biological systems and by fostering greater interaction and collaboration among plant and chemical biologists.

\section{Existing challenges}

An immediate challenge for budding plant chemical geneticists is selecting the right chemical library for a plant-based chemical screen. Conventional libraries that are frequently used in chemical screens include fragment-based libraries, focused libraries, approved drug collections, and unbiased collections. Many chemical libraries, such as ChemBridge DIVERSet, Analyticon Discovery, and Prestwick Library etc., are available commercially ${ }^{1}$. Compounds are generally dissolved in a suitable solvent, such as dimethyl sulfoxide and are supplied in 96-well or 384-well formats. The libraries are easily accessible and can be utilized extensively by plant biologists ${ }^{1}$, but most of them are inclined towards targeted screens used in mammalian cell culture assays and are designed according to mammalian cells absorption, distribution, metabolism, and excretion (ADME) criteria, and to adhere to the Lipinski's Rule of Five ${ }^{1}$. Thus, such libraries might be less suited to yield bioactive compounds for intact plants. Using high concentrations for plant screens can overcome some uptake issues, but, at the same time, may impart unwanted toxic or non-specific effects in plants. Besides the suitability of the libraries for plants, a rather different obstacle lies in the high cost of these chemical collections which might limit their use by individual labs.

The design and choice of chemical screening phenotypes, access to specialized highthroughput screening facilities and proper automation for rapid analysis are some of the key impediments that require attention. Initially, the discovery of small molecules in plants was mainly centered on qualitative forward chemical genetic screens which scored phenotypes 
resembling known hormonal treatments or existing mutants such as hypocotyl and root lengths, lateral root numbers, venation patterns, or endomembrane trafficking perturbations ${ }^{1}$. While such chemical screens delivered interesting and potentially useful candidate hits, the resulting target validation revealed that the observed phenotypes were often due to alterations in diverse biological pathways, beyond what was predicted, making the effects less specific ${ }^{1}$. Therefore, development of well-designed and pathway-focused scoring and screening systems using in vivo selective marker and gene-reporter assays need to be employed to assess whether a particular signaling pathway is altered. The discovery of jarin-1 as a jasmonate signaling inhibitor was a result of such a strategy $(\text { Fig. 2a })^{9}$. The availability of jasmonate-responsive reporters facilitated an easy and focused phenotypic screening followed by validation and target identification based on the plethora of available knowledge regarding jasmonate signaling components and their biological activities. Employing such pathway-focused screens would assist in the elimination of compounds with broader and non-specific effects.

Plant researchers also face the challenge that they currently have limited access to traditional 'medicinal chemistry' approaches commonly used to probe the mechanism of small molecule action. As an example, after identifying a hit from a screen, an inexperienced plant chemical geneticist may proceed towards target identification with a non-optimized hit compound. This overlooks the important structure-activity relationship analysis and compound hit optimization steps, including potency and selectivity assays that are normally done in pharmaceutical research. As a result, these non-optimized hit compounds have low target affinities or potential cellular uptake liabilities, which hinder their use in the downstream targetidentification process and limit their applicability as chemical probes in plants. There are many potential reasons for this jump to target identification stages. Firstly, a typical plant chemical 
genetic project from conceptualization to target identification and designing new effective analogs, which if not available from commercial sources, is time-consuming. In addition, the costs of medicinal chemistry involved in creating such analogs can run high which may demotivate some project leaders working on tight budgets. Finally, the lack of suitable chemical infrastructure in plant research centers can be a major impediment.

While the above-mentioned factors stand in the way of plant biologists adopting a chemical genetic mindset in their research, there are promising signs of progress.

\section{Changing the mindset}

For a plant biologist, the ability to grasp concepts of other scientific disciplines such as chemistry that are needed for expertise in chemical genetic approaches may be uncomfortable at first. However, the next generation of plant biologists needs to broaden their horizons, and this begins with early training. Young plant scientists should be encouraged and supported to attend training programs focused at the intersection between chemistry and biology. The European Molecular Biology Organization (EMBO) chemical biology training programs are notable in this regard. Similarly, early-stage plant scientists interested in chemical genetics should participate and attend cross-disciplinary chemical biology conferences to interact with a diverse group of participants from chemistry departments and pharmaceutical companies to foster collaborations and learn about new and emerging chemical tools. In this regard, the development of focused plant chemical biology conferences or symposiums will encourage plant chemical geneticists to present their research work, learn from experts, and foster collaboration with industrial partners. Furthermore, plant biologists should routinely enter into discussions and collaborations with 
chemists or biophysicists who have a shared goal of advancing mechanistic understanding in plant science.

Changing our mindset on how to design and implement chemical screens is another route to consider. Recently, there has been a growing trend of switching from phenotypic chemical genetic screens towards target-based or reverse chemical genetic approaches (Fig. 2b). For example, NPD12704 was recently identified in a target-based screen as the first peptide-hormone signaling inhibitor in Arabidopsis ${ }^{14}$. In this work, a receptor kinase of choice, BAM1, was immobilized on beads and subjected to a chemical library to identify compounds that interfered with the binding of BAM1's peptide ligand, CLE9. In this way, only receptor-specific compounds were identified, thereby avoiding off-targets. Shifting from a phenotypic to targetbased approach can be further complemented by the advancements in NMR-based screening, structural biology, in silico analyses, and three-dimensional structural modeling of proteins. Consequently, we are now in a position to apply this knowledge to modify either or both endogenous ligands and their protein receptors to perform targeted chemical genetics approaches (Fig. 2c, d) ${ }^{15-17}$ For example, modification of the endogenous jasmonoyl-isoleucine ligand has been shown to selectively activate the JAZ9-EIN3/EIL1-ORA59 signaling branch of jasmonate signaling ${ }^{15}$. Similarly, a modified fluorogenic strigolactone agonist, Yoshimulactone Green, has proven useful to study functions of the Arabidopsis D14-type strigolactone receptors and to dissect the signal perception mechanism of strigolactone receptors in the parasitic plant Striga ${ }^{16}$. Also, in a slightly different approach based on the crystal structure of the TIR1-IAA-AUX/IAA complex of auxin signaling, an orthogonal small molecule-based system has been designed to produce a modified "convex auxin" (cvxIAA) that binds only to an altered receptor (ccvTIR1). 
This system eliminates interference with endogenous IAA-TIR 1 signaling ${ }^{17}$ and bypasses genetic redundancy and feedback regulations.

In light of the success stories outlined above, plant biologists should feel motivated to utilize the structural information available for their proteins of interest and collaborate with $\mathrm{X}$ ray crystallographers and chemoinformatics experts to employ in silico methods as a general way of identifying novel binding sites in their protein of interest as well as within the whole plant proteome (Fig. 3). While structural modeling provides an initial start, detailed cellular and target identification studies are still needed to confirm and validate small molecule-target interactions, so plant biologists also need to strengthen their connections with chemical biologists.

\section{Tackling target identification}

Classical genetics has greatly enabled target identification for small-molecule compounds in plants. The most common approach involves analyzing the biological activity of a compound across a mutagenized seed population to link activity perturbations to potential molecular targets using genome sequencing and mapping ${ }^{1,2,18}$. However, classical genetics as a means of target identification is limited in cases where the target is an essential gene, which may cause embryonic lethality when mutated. As a result, new approaches are needed to advance target identification in plants.

While chemical proteomics has proven to be a powerful tool in drug discovery research ${ }^{18}$, only a few labs have successfully utilized it in plants ${ }^{19-22}$ as such approaches generally require extensive chemical probe synthesis. In such scenarios, plant biologists should actively collaborate with organic chemists to design improved analogs with enhanced bioactivity or affinity probes derived from the bioactive compound to allow target identification methods. (Fig. 
3). In addition, they should also develop inactive structural analogs of their chemical probes to use as negative control compounds in their experiments.

Plant biologists could also consider certain biochemical methods, such as the drug affinity responsive target stability (DARTS) and cellular thermal shift assay (CETSA), in which compound modification is not required for target identification and validation ${ }^{18}$. For instance, the small molecule ES9-17, a clathrin-mediated endocytosis inhibitor, was recently validated to bind to its target, clathrin heavy chain, via DARTS and CETSA ${ }^{21}$. An inherent advantage of using these methods lies in their complementation with advanced mass spectrometry and transcriptomic methods to study the impact of a small molecule at a global proteome or genome level (Fig. 3). Given that such core facilities are generally accessible to plant biologists, they should not feel confident in undertaking chemical genetics projects from this perspective. It is important to realize that one of the main advantages of studying the mode-of-action and identifying protein targets of bioactive compounds is the high probability of detecting proteins with unannotated function as well as uncovering novel functions of a protein in additional signaling pathways.

\section{Bridging the gaps}

In the "Existing challenges" section, we discussed issues with accessing chemical libraries for plant-based screens. One way to tackle this problem is the establishment of inter-laboratory screening "core" facilities with access to useful chemical libraries for all the participating laboratories. Many research institutes may already possess annotated compound collections that can be easily accessed for possible chemical biology projects, often for external investigators. As 
these chemical libraries may have been successful in screenings of other model organisms, the potential for an abundant amount of cross-disciplinary knowledge might prove worthwhile.

Current chemical libraries designed for mammalian screening may not be sufficient, given the presence of a cell wall in plants, which might affect compound permeation. Arabidopsis seedlings can easily fit in 24 to 96 well plates ${ }^{1,2}$ and can be grown relatively inexpensively in either solid or liquid growth media to screen compounds. To this end, the use of liquid media, which in addition to providing high compatibility with automation can also assist in answering the key concern of compound bioavailability. Most likely, only those compounds that can permeate the cell-wall barrier shall be able to modulate a biological response in the cell, although responses initiated at the cell-wall level can also be interesting.

Another approach could be collaborating with chemical biologists to create new chemical libraries suited for screening in plants that may enhance success and address the shortcomings of current libraries. Thankfully, for aspiring plant chemical geneticists, certain plant-focused compound collections are available: LATCA (Library of AcTive Compounds on Arabidopsis) and Plasma Membrane Recycling Set A and Set B (PMRA/B), which were assembled from different libraries and have been successfully tested on plants ${ }^{1}$. Hence, plant biologists who utilize such libraries instead of completely unannotated chemical libraries can be more assured of discovering compounds with a biological effect and some initial knowledge about their mode-ofaction.

Entering into industrial collaborations is a unique way to access chemical space that may not be widely available (Fig. 3). The pharmaceutical and agrochemical companies are excellent sources of compound collections because they routinely synthesize and test new chemical scaffolds. Over the years, detection of compounds with a new mode-of-action, searching for new 
druggable targets, and overcoming small-molecule resistance have become key challenges for the industry. Not surprisingly, many collaborations between pharmaceutical companies and academia have been implemented after notable successes have provided tremendous benefits for both communities ${ }^{23}$. For the plant world, a few promising initiatives such as the AGRI-net chemical biology society and EU-OPENSCREEN have started to bring together industrial and academic communities together to develop novel research tools for plant sciences ${ }^{24}$. The possibility that teaming up with an industrial partner on a chemical genetics project may convert an idea into a tangible marketable product should push plant scientists to seek industrial collaborations. In such cases, early negotiations around issues of patent protection and intellectual property would ensure fruitful collaborations.

\section{Final perspectives}

Chemical genetics has been useful in addressing diverse biological questions but the potential to apply this approach towards emerging fields in plant biology remains tantalizing. The implementation of recent advances in chemical biology such as bio-orthogonal chemistry ${ }^{17}$, fluorescently labeled peptide ligands ${ }^{14}$ and optogenetic probes ${ }^{25}$ have a strong potential to mitigate certain limitations of classical plant genetics.

We propose a holistic approach toward increasing awareness about chemical genetics within the plant science world. Visibility of plant chemical genetics needs to be increased at international plant biology conferences and in scientific journals. At the same time, we need to provide appropriate training to early career plant biologists in areas such as chemical screenings, chemical probe development, application, and target identification. 
In addition to instrument automation, improved microscopy, and plant-based chemical library accessibility, plant biologists need more venues to discuss their findings. This could be facilitated through annual plant chemical genetics workshops or online platforms where expert plant chemical geneticists share knowledge and resources to help early-stage researchers in their chemical genetics campaigns. We hope that plant biologists will make a concerted effort to work more closely with chemical biologists and adopt a chemical genetics mindset to advance the frontiers of plant biology research in the coming decade. 


\section{References}

1. Dejonghe, W. \& Russinova, E. Plant Physiol. 174, 5-20 (2017).

2. Hicks, G. R. \& Raikhel, N. V. Front. Plant Sci. 5, 455 (2014).

3. Bucci, M., Goodman, C. \& Sheppard, T. L. Nat. Chem. Biol. 6, 847-854 (2010).

4. Park, S.-Y. et al. Science 324, 1068-1071 (2009).

5. Drakakaki, G. et al. Proc. Natl. Acad. Sci. USA 108, 17850-17855 (2011).

6. Halder, V. et al. Sci. Rep. 9, 11196 (2019).

7. He, W. et al. Plant Cell 23, 3944-3960 (2011).

8. Tsuchiya, Y. et al. Nat. Chem. Biol. 6, 741-749 (2010).

9. Meesters, C. et al. Nat. Chem. Biol. 10, 830-836 (2014).

10. De Rybel, B. et al. Chem. Biol. 16, 594-604 (2009).

11. Bektas, Y. \& Eulgem, T. Front. Plant Sci. 5, 804 (2015).

12. Brabham, C. \& DeBolt, S. Front. Plant Sci. 3, 309 (2013).

13. Yoneda, A. et al. Plant Cell Physiol. 48, 1393-1403 (2007).

14. Shinohara, H. et al. Commun. Biol. 2, 61 (2019).

15. Takaoka, Y. et al. Nat. Commun. 9, 3654 (2018).

16. Tsuchiya, Y. et al. Science 349, 864-868 (2015).

17. Uchida, N. et al. Nat. Chem. Biol. 14, 299-305 (2018).

18. Dejonghe, W. \& Russinova, E. Front. Plant Sci. 5, 352 (2014).

19. Zhang, C. et al. Proc. Natl. Acad. Sci. USA 113(1):E41-50 (2016).

20. Mishev, K. et al. Plant Cell 30, 2573-2593 (2018).

21. Dejonghe, W. et al. Nat. Chem. Biol. 15, 641-649 (2019).

22. Uehara, T.N. et al. Proc. Natl. Acad. Sci. USA 116(23):11528-11536 (2019).

23. Mallapaty S. Nature 552, S5 (2017). 
24. Meiners, T., Stechmann, B. \& Frank, R. J. Chem. Biol. 7, 113-118 (2014).

25. Papanatsiou, M. et al. Science 363(6434):1456-1459 (2019).

\author{
Affiliations \\ Vivek Halder and Eugenia Russinova are in the Department of Plant Biotechnology and \\ Bioinformatics, Ghent University, 9052 Ghent, Belgium and the Center for Plant Systems \\ Biology, VIB, 9052 Ghent, Belgium. \\ Current address for VH: Rijk Zwaan, De Lier, 2678 ZG, The Netherlands \\ E-Mail: eurus@psb.vib-ugent.be
}

\title{
Acknowledgments
}

We thank Natasha Raikhel, Glenn R. Hicks, Tom Beeckman, Markus Kaiser, Dominique

Audenaert, Wim Dejonghe and Kiril Mishev for critical reading and valuable suggestions, and Martine De Cock for help in preparing the manuscript. This work was supported by the Research Foundation-Flanders (project 3G009018 to E.R.).

\section{Competing financial interests}

The authors declare no competing financial interests.

\section{Figure legends}

Figure 1: The balance between classical genetics and chemical genetics for probing mechanisms in plant biology. Classical genetics (left side), which involves mutagenizing plants and mapping 
the relevant genes that cause the mutant phenotype and has historically served as the primary study method in basic plant research. Chemical genetics (right side) involves the application of a small molecule that resembles genetic mutations of the protein-encoding gene phenotypically.

Figure 2: Conventional and current trends in plant chemical genetics (a) Forward chemical genetics, illustrated by jarin-1 that targets the JAR1 enzyme in the JA-signaling pathway, involves a qualitative or quantitative phenotypic screening followed by compound characterization and protein target identification (b) Reverse chemical genetics, exemplified by NPD12704 that targets the Arabidopsis receptor kinase BAM1 and interferes with the binding of its cognate peptide ligand CLE9, is a relatively recent trend in plant chemical genetics which involves screening of a chemical library against a purified protein of interest. (c, d) Another variant of reverse chemical genetics involves modifying endogenous ligands such as the modified strigolactone, yoshimulactone green, which binds to strigolactone D-14 receptor; or the modified jasmonate, jasmonoyl-isoleucine, which can selectively activate JAZ9-EIN3/EIL1ORA59 signaling. A slightly different approach involves modification of both endogenous ligand and its receptor as seen in the case of modified convex auxin (cvxIAA) that selectively binds to its modified concave receptor (cvvTIR1).

Figure 3: Plant chemical genetics will benefit from cross-disciplinary approaches. To broaden the scope of plant chemical genetics, plant biologists need to harness the advances in the fields of chemistry, proteomics, transcriptomics, computational biology, crystallography, and collaborate with academic and industrial partners. 